\title{
Special Online Issue Dedicated to Translation Research and Biomarkers: Author Disclosure Summary
}

The authors of articles included in this special online issue disclosed upon submission of their articles the commercial funding that may have supported the research reported. In addition, upon selection of their article to be included in this special issue, authors were then requested to answer the following questions:

1. Did you or your institution at any time receive payment or services from a third party for any aspect of the submitted work (including but not limited to speaker bureau, grants/research support, consultant, stock shareholder [directly purchased], employee)? (Y/N)

2. If Yes for any of the above, please provide the name of the entity.
- Speaker Bureau:

- Grants/Research Support:

- Consultant:

- Stock Shareholder (directly purchased):

- Employee:

- Other Relevant Commercial Payment or Service Received:

In response to this query, the following authors provided details regarding support received. All other authors had nothing to disclose.

\begin{tabular}{|c|c|c|c|}
\hline DOI & Title & $\begin{array}{l}\text { Corresponding } \\
\text { author }\end{array}$ & Disclosure \\
\hline $10.1245 / \mathrm{s} 10434-011-1881-2$ & $\begin{array}{l}\text { Inhibition of Heat Shock Protein Response } \\
\text { Enhances PS-341-Mediated Glioma Cell } \\
\text { Death }\end{array}$ & S. Zhao & $\begin{array}{l}\text { Grant support: National Natural Science } \\
\text { Foundation, China }\end{array}$ \\
\hline $10.1245 / \mathrm{s} 10434-011-2123-3$ & $\begin{array}{l}\text { 3'-UTR Displays a Correlation with the Risk } \\
\text { and Clinicopathological Development of } \\
\text { Hepatocellular Carcinoma }\end{array}$ & Chia-Jui Weng & $\begin{array}{l}\text { Grant support: the National Science Council, } \\
\text { NSC99-2313-B-165-001-MY3, Taiwan, } \\
\text { Republic of China }\end{array}$ \\
\hline 10.1245/s10434-011-1825-x & $\begin{array}{l}\text { Prognostic Significance of TWEAK } \\
\text { Expression in Colorectal Cancer and Effect } \\
\text { of Its Inhibition on Invasion }\end{array}$ & $\begin{array}{l}\text { Cheng-Chi } \\
\text { Chang }\end{array}$ & $\begin{array}{l}\text { Grant support: National Science Council, } \\
\text { Taiwan (NSC 97-2314-B-002-031-MY2) }\end{array}$ \\
\hline $10.1245 / \mathrm{s} 10434-011-1970-2$ & $\begin{array}{l}\text { Claudin-4 Expression Predicts Survival in } \\
\text { Pancreatic Ductal Adenocarcinoma }\end{array}$ & Norihiro Sato & $\begin{array}{l}\text { Grant support: Ministry of Education, Culture, } \\
\text { Sports, Science and Technology of Japan }\end{array}$ \\
\hline $10.1245 / \mathrm{s} 10434-011-2040-5$ & $\begin{array}{l}\text { Increased CD13 Expression Reduces } \\
\text { Reactive Oxygen Species, Promoting } \\
\text { Survival of Liver Cancer Stem Cells via an } \\
\text { Epithelial-Mesenchymal Transition-like } \\
\text { Phenomenon }\end{array}$ & $\begin{array}{l}\text { Hideshi Ishii } \\
\text { and Masaki } \\
\text { Mori }\end{array}$ & $\begin{array}{l}\text { Grant support: Core Research for Evolutional } \\
\text { Science and Technology (CREST), a Grant- } \\
\text { in-Aid for Scientific Research on Priority } \\
\text { Areas (20012039), Grants-in-Aid for } \\
\text { Scientific Research S (21229015) and C } \\
\text { (20590313) from the Ministry of Education, } \\
\text { Culture, Sports, Science, and Technology, } \\
\text { and a grant from the Tokyo Biochemical } \\
\text { Research Foundation, Japan }\end{array}$ \\
\hline $10.1245 / \mathrm{s} 10434-011-2043-2$ & $\begin{array}{l}\text { Prognostic Significance of NBS1 and Snail } \\
\text { Expression in Esophageal Squamous Cell } \\
\text { Carcinoma }\end{array}$ & $\begin{array}{l}\text { Liang-Shun } \\
\text { Wang }\end{array}$ & $\begin{array}{l}\text { Grant support: National Science Council } \\
\text { (NSC95-2314-B-303-023) and Tzu Chi } \\
\text { Foundation Medicine Mission } \\
\text { (TCRD-I9603-03) }\end{array}$ \\
\hline $10.1245 / \mathrm{s} 10434-012-2216-7$ & $\begin{array}{l}\text { Cell Cycle Proteins Predict Recurrence in } \\
\text { Stage II and III Colon Cancer }\end{array}$ & Eric Belt & $\begin{array}{l}\text { Grant support: Posthumus Meyjes Research } \\
\text { Foundation of the Kennemer Gasthuis } \\
\text { Hospital, Haarlem, The Netherlands }\end{array}$ \\
\hline
\end{tabular}

